\title{
P27 Non-validated Blood Pressure Devices Dominate the Online Marketplace: An Initiative of the Lancet Commission on Hypertension Group
}

Dean Picone ${ }^{1}$, Rewati Deshpande ${ }^{1}$, Martin Schultz ${ }^{1}$, Ricardo Fonseca ${ }^{1}$, Norm Campbell ${ }^{3,4}$, Christian Delles ${ }^{5}$, Michael Hecht-Olsen ${ }^{6,7}$, Aletta Schutte ${ }^{8}$, George Stergiou ${ }^{9}$, Sonia Angell ${ }^{10}$, Raj Padwal ${ }^{11}$, James Sharman ${ }^{1}$

\author{
${ }^{1}$ Menzies Institute for Medical Research, College of Health and Medicine, University of Tasmania, Australia, Australia \\ ${ }^{3}$ Department of Medicine, Physiology and Pharmacology and Community Health Sciences, O'Brien Institute for Public Health, Calgary, Canada \\ ${ }^{4}$ Libin Cardiovascular Institute of Alberta, University of Calgary, Calgary, Alberta, Canada \\ ${ }^{5}$ Institute of Cardiovascular and Medical Sciences, University of Glasgow, Glasgow, UK \\ ${ }^{6}$ Department of Internal Medicine, Holbaek Hospital, Holbaek, Denmark \\ ${ }^{7}$ Centre for Individualized Medicine in Arterial Diseases (CIMA), Odense University Hospital, University of Southern Denmark \\ ${ }^{8}$ Hypertension in Africa Research Team, Medical Research Council Unit for Hypertension and Cardiovascular Disease, \\ North-West University, Potchefstroom, South Africa \\ ${ }^{9}$ Hypertension Center STRIDE-7, National and Kapodistrian University of Athens, School of Medicine, Third Department of Medicine, \\ Sotiria Hospital, Athens, Greece \\ ${ }^{10}$ Division of Prevention and Primary Care, New York City Department of Health and Mental Hygiene, New York, USA \\ ${ }^{11}$ Department of Medicine, University of Alberta, Edmonton, Alberta, Canada
}

\section{ABSTRACT}

Introduction: Home BP monitoring is recommended to guide clinical decisions on hypertension and is used worldwide. People make their own decisions when purchasing BP measurement devices, which are often made online. One potential barrier to accurate home BP monitoring is that patients may purchase an unvalidated device (one that has not been proven accurate according to an internationally sanctioned protocol). This study aimed to evaluate the number, type, percentage validated and cost of home BP devices available online.

Methods: A systematic search of online businesses selling BP devices that may be used for home BP monitoring was conducted. Multinational companies make international deliveries, so searches were restricted to BP devices available for one nation (Australia) as an example of device availability through the globally connected online marketplace. Validation status of BP devices was determined according to established protocols.

Results: 59 online businesses, selling 972 unique BP devices were identified. These included 278 upper-arm cuff devices (18.3\% validated), 162 wrist-cuff devices ( $8.0 \%$ validated) and 532 wrist-band wearables ( $0 \%$ validated). Most BP devices $(92.4 \%)$ were stocked by international 'e-commerce' businesses (e.g. eBay, Amazon), but only $5.5 \%$ of these were validated. Validated cuff BP devices were more expensive than non-validated devices: median (interquartile range) of 101.14 (75.00 to 151.50; versus 67.37 (30.40 to 112.83) AUD, $p<0.0001$.

Conclusion: Non-validated BP devices dominate the online marketplace and are sold at lower cost than validated devices. The widespread use of non-validated BP devices is a barrier to accurate home BP monitoring and must be urgently addressed.

(C) 2019 Association for Research into Arterial Structure and Physiology. Publishing services by Atlantis Press International B.V. This is an open access article distributed under the CC BY-NC 4.0 license (http://creativecommons.org/licenses/by-nc/4.0/). 